\title{
A Critical Examination of Cases Closed After Application for VR Services
}

\author{
Kira Hollywood ${ }^{1}$ a \\ 1 Texas Workforce Commission-Vocational Rehabilitation Division \\ Keywords: vocational rehabilitation, application, case closure \\ https://doi.org/10.52017/001c.27919
}

\section{Rehabilitation Counselors and Educators Journal}

Vol. 10, Issue 2, 2021

\begin{abstract}
In the Texas Combined (Blind and General) Vocational Rehabilitation Services Division, $15 \%$ of cases were closed after an application was taken, but before eligibility was determined during the September 1, 2018 to August 31, 2019 time frame. This study was conducted to identify the primary reasons for these closures. Case services data and vocational rehabilitation (VR) counselor case notes were analyzed to determine if any substantive trends related to case closure after application were present and identify potential factors indicating a higher risk of case closure for those in application status. Further, the investigation found (a) errors in reporting codes by VR counselors, (b) individuals with intellectual and psychological disabilities being closed prematurely at higher rates than those with physical or other disabilities, and (c) a notable absence of communication from VR counselors informing applicants that their case was being closed. The findings from this study support the need for further assessment of why cases are closed after an application for VR services and indicate the need for VR staff training to clarify agency processes and participant engagement strategies to address and prevent premature closure of cases.
\end{abstract}

\section{Background and Purpose}

In $2019,38.9 \%$ of individuals with disabilities aged 18-64 years were employed, compared with $78.6 \%$ of people without disabilities (Houtenville \& Rafal, 2020). The public Vocational Rehabilitation (VR) program is an important statefederal partnership designed to help address this disparity and improve employment rates for individuals with disabilities. In the 2019 program year, 361,684 individuals applied to the VR program nationally, and 93\% were determined eligible for services (Pope, 2020). While these figures illustrate a large and robust public disability program, VR has experienced a downward trend in recent years, with the number of individuals applying for services decreasing nearly $25 \%$ between 2017 and 2019.

Prior research has identified poor counselor-consumer relationships, lack of transportation, severity of disability, lack of work history, geographic location, and consumer dissatisfaction in the VR program as contributing factors to unsuccessful closures (Ipsen \& Swicegood, 2014; Migliore \& Landa, 2017; Park et al., 2005; Rogers et al., 2011). While many of these studies focus on case closure after an individual plan for employment (IPE) is written, a longitudinal study by RTI International (2003) identified certain consumer characteristics that influenced case closure prior to eligibility determination, including, age, educational sta- tus, marital status, work history and earnings, and careerrelated interests. Variables that predicted whether or not an individual would be eligible for VR services following application included (a) whether an individual's disability was noted as significant or most significant, (b) intellectual disability or hearing impairment, (c) higher self-esteem, and (d) consumer employment at the time of application (RTI International, 2003). However, given the study was conducted nearly 20 years ago, it is unclear whether these variables remain predictive today.

The Texas Workforce Commission-Vocational Rehabilitation Division (TWC-VR) monitors case closures and seeks to better understand the circumstances surrounding the higher number of cases closed after application. According to data obtained through the Texas Workforce CommissionDivision of Operational Insight (DOI), during the first three quarters of Fiscal Year (FY) 2019 (September 1, 2018-July $1,2019)$, there were 4,035 cases closed after application, which accounted for $15 \%$ of all applications taken during that time frame. This represents an early closure rate double the national average of $7 \%$ (Pope, 2020). Premature case closure following application is a concern, as the reasons may not entirely reflect ineligibility or lack of consumer interest in participating, but rather may represent process errors within the control of VR agencies to correct.

The Rehabilitation Services Administration (RSA) re- 
quires VR agencies to document and report closure reasons for cases closed after application through the Reporting Manual for the Case Service Report (RSA-911). Reasons for case closure must be identified by a Vocational Rehabilitation Counselor (VRC) and recorded using prescribed codes and VR agencies policy. Furthermore, the Vocational Rehabilitation Services Manual (VRSM) states that an individual must be notified of case closure, and "written notification must be sent by letter or encrypted email at least 10 business days prior to closing the case, to allow time for the customer to contact the VR counselor if there are any concerns about closing the case" (VRSM B-605: Customer Notification).

The purpose of this study was to investigate why cases were closed for individuals who applied for VR services prior to an eligibility determination through the TWC-VR program. The evaluation questions posed were as follows:

1. What are the primary reasons for early case closure?

2. Did the data coding for early closure align with VRC case notes?

3. Were consumers contacted prior to case closure?

4. Which disability types were represented in the early case closure data?

\section{Methods}

Case services data was obtained internally through the Texas Workforce Commission- DOI for the September 1, 2019 through February 28, 2020 time frame on individuals who had applied for VR services through TWC-VR and whose cases were closed after application, but prior to eligibility determination. The dataset contained the consumer's name, the region and unit office in which their case was located, self-reported Supplemental Security Income and/ or Social Security Disability Insurance (SSI/SSDI) benefits, self-reported veteran status, reason for closure, and the date the application was taken. This timeframe was chosen as it provided the most recent data available, and trends for this timeframe were consistent with case closure data for the prior two years. Quantitative data analysis was used to identify factors that may indicate a higher risk of case closure for those in application status. Using frequency analysis, the primary reasons for case closure were then compiled for review. To provide a more in-depth analysis, cases were individually reviewed for each of the top three closure reasons to determine if closure reasons selected by VRCs aligned with the closure code selected.

Using systematic random sampling, twenty-four (24) cases were selected for each closure reason by choosing the first 12 cases listed in each quarter (12 cases in quarter one, and 12 cases in quarter two). Sampling was spread equally over the state based on regional population size. The cases were analyzed to see whether reasons for case closure coded by the VRC aligned with their case notes regarding the actual reason for case closure. Other factors included whether the individual was contacted at closure, contacted at any time between the application for VR services and closure, whether an extension of time (EOT) was used in the case, or if the individual was placed in Pre-Eligibility Trial Work Services. Extensions of time are used when there are "exceptional and unforeseen circumstances” (34 CFR §361.41) that result in the VR counselor requiring longer than 60 days for an eligibility determination to occur.

Pre-Eligibility Trial Work Services are used if there is a question regarding whether an individual will be able to become successfully employed in a competitive, integrated work setting. A trial work plan is written, and the individual is required to participate in at least three trial work activities in real-work settings with a contracted provider. VR counselors have a maximum of 12 months to determine if the individual can achieve an employment goal in an integrated (non-sheltered workshop) setting.

Data from case notes was also analyzed regarding the individual's reported disability at application. Disability groups were categorized into the seven codes reported to RSA (a) hearing impairment, (b) visual impairment, (c) psychosocial/psychological impairment, (d) physical impairment, (e) intellectual disability, (f) learning disability, and (g) no disabling condition.

\section{Results}

The data provided from Texas Workforce Commission DOI identified a total of 1,897 cases closed between September 1, 2019 and February 28, 2020 without eligibility determination. The three most frequent reasons (representing 73\%) for case closure following application, but prior to plan development, were identified as (a) no longer interested in receiving services or further services (34\%), (b) all other reasons (24\%), and (c) unable to locate or contact after three or more documented attempts (15\%). See Table 1.

\section{No Longer Interested in Receiving Services or Further Services}

Closure coded as No Longer Interested in Receiving Services was the primary reason noted, representing $34 \%$ of all cases closed unsuccessfully following application for services. This option should be selected only when an individual has clearly indicated they no longer wish to actively participate in VR services. Review of the 24 cases randomly selected from the quarterly dataset were then compared with VRC case notes to evaluate consistency regarding reason for closure, the individual's reported disability at application, whether the individual was contacted at any time between the application for services and closure, whether the individual was informed of the closure, and whether the individual's case was entered into an extension of time or put into pre-eligibility trial work.

Case notes indicated the majority of individuals (79\%) were contacted at least once after the application was taken. Most individuals (79\%) were also informed that their case was going to be closed. However, $21 \%$ of individuals were never contacted after their application for VR services, and similarly, $21 \%$ of individuals were not informed of their case being closed. In 14 (58\%) of the cases examined, a match between the case notes and coded data indicated the reason for closure as the individual was no longer interested in receiving services. Reasons varied, with some not wishing to obtain employment at the time and/or no longer needing VR services, whereas others had obtained employment on their own. However, errors were identified in 10 of the 24 
Table 1. TWC-VR Reasons for Case Closure After Application FY 2020

\begin{tabular}{|c|c|c|}
\hline Reasons for closure after application & \# of cases closed & Percent \\
\hline All other reasons & 454 & $24 \%$ \\
\hline Criminal offender & 8 & $<1 \%$ \\
\hline Currently not legally able to work in the U.S. & 19 & $1 \%$ \\
\hline Death of individual & 1 & $<1 \%$ \\
\hline Does not require VR service & 80 & $4 \%$ \\
\hline Health/Medical & 48 & $3 \%$ \\
\hline Ineligible & 90 & $5 \%$ \\
\hline Ineligible - pursuant to section 511 & 4 & $<1 \%$ \\
\hline No disabling condition & 96 & $5 \%$ \\
\hline No impediment to employment & 155 & $8 \%$ \\
\hline No long-term source of extended services available & 1 & $<1 \%$ \\
\hline No longer interested in receiving services or further services & 636 & $34 \%$ \\
\hline Not available for services; in institutional setting other than prison/jail & 7 & $<1 \%$ \\
\hline Transferred to another agency & 10 & $1 \%$ \\
\hline Transportation not feasible & 5 & $<1 \%$ \\
\hline Unable to locate or contact (after three or more documented attempts to contact) & 283 & $15 \%$ \\
\hline
\end{tabular}

cases (42\%), where closure reasons had been coded inaccurately.

Regarding the closure reason of No Longer Interested in Receiving Services and types of disability, of the 24 cases reviewed, 16 (67\%) individuals were identified as having intellectual disabilities, which was substantially higher than all other disability categories. Fifty percent $(50 \%)$ of all sampled cases in this area were listed as receiving SSI/SSDI at some point during the life of the case; however only $8 \%$ of cases overall coded as No Longer Interested in Receiving Services were coded similarly. For cases in which the individual was receiving SSI/SSDI, there was limited or no documentation in the VRC case notes related to discussing SSI/SSDI work incentives options and/or whether they may be interested in participating in the Ticket to Work program offered through the Social Security Administration.

Of interest were the number of cases listed as No Longer Interested in Receiving VR Services and those in Pre-Eligibility Trial Work. Fourteen (14) out of 24 cases (58\%) were listed as "yes" for Pre-Eligibility Trial Work. In almost all of these cases, VRC case notes were consistent with this assignment. Subsequently, it was decided to review another 12 cases previously closed for the same reason. Of these cases, however, only one was listed for Pre-Eligibility Trial Work. It was not possible to determine how many cases overall were listed as pre-eligibility trial work with the case closure reason of No Longer Interested in Receiving Services without reviewing each case individually. Statewide, there were on average around 100 cases in Pre-Eligibility Trial Work in Texas for FY 2020. While this does not represent a large number, these cases need to be monitored closely to ensure individuals have fair opportunities to pursue work in competitive, integrated settings.

\section{All Other Reasons}

The second highest reason noted was All Other Reasons, accounting for $24 \%$ of early case closures. This code is to be used when none of the existing TWC-VR "Reasons for Case Closure After Application” apply. A VRC might select this code when an individual does not provide the necessary documentation to move forward with eligibility determination, or when an individual obtains employment through another agency without informing VR staff. VR counselors are instructed to provide thorough information in the VRC case notes regarding exactly why the case was closed to ensure the actual reason is clear, and that other closure reasons were not appropriate for the case.

This closure reason revealed the highest rate of individuals not being contacted prior to case closure, or never being contacted at all between application and closure. Seventyone $(71 \%)$ of cases reviewed had no case notes entered indicating the individual was informed of case closure. Fifty percent $(50 \%)$ of cases reviewed had no case notes indicating the individual was ever contacted between the time of application and case closure. This implies the VRC may have only spoken to the individual at application, did not contact them thereafter, and did not inform them of their impending case closure. If the counselor did attempt to contact the individual, it was not recorded or reflected in the case notes.

The majority of cases listed as All Other Reasons were primarily due to the individual not providing required documentation or attending appointments necessary for the VR counselor to make a correct eligibility determination as illustrated in Table 2.

Disability reported by the individual at application was highest for those with intellectual disabilities, with 9 cases (37\%) in this disability category. The majority of reasons noted were the VR counselor was unable to obtain records, 
Table 2. Reasons for Case Closure Based on Case Note Information-All Other Reasons

\begin{tabular}{|c|c|}
\hline Identified reasons for case closure based on case note information & \# of cases $(\%)$ \\
\hline Closed because a doctor's appointment could not be scheduled & $1(4 \%)$ \\
\hline Individual did not provide required documents or attend appointments necessary for determining eligibility & $8(33 \%)$ \\
\hline Disability too severe & $2(8 \%)$ \\
\hline Health/Medical & $1(4 \%)$ \\
\hline No longer interested in services & $1(4 \%)$ \\
\hline No reason listed & $1(4 \%)$ \\
\hline Unable to locate & $4(17 \%)$ \\
\hline Unclear- Not indicated in case notes & $6(25 \%)$ \\
\hline Total & 24 \\
\hline
\end{tabular}

the case was coded incorrectly and another reason for closure should have been selected, or the individual was actually unable to locate before closure.

\section{Unable to Locate}

The third highest reason for case closure were cases coded as Unable to Locate. This closure code is to be used when an individual has relocated or has not responded to three or more contact attempts via mail, telephone, text, or email. In these circumstances, case closure should only occur after a minimum of three attempts to contact the individual have occurred, and include sending written notification to the individual 10 business days before closing the case. Ten (42\%) cases reviewed met the criteria needed to use the Unable to Locate closure code. Other explanations listed in VRC case notes were that the individual did not provide required documents for eligibility determination, there was no documentation the VRC attempted to contact the individual before closing the case, or the individual was no longer interested in receiving services.

While it is understandable that in some cases, the individual may not be available for contact, 8 out of 24 cases (33\%) indicated that contact with the individual was never attempted following application. This trend can be seen across all three closure reason categories and is concerning, given that individuals who may have wanted to engage in VR services lost the opportunity to do so at that point in time. Additionally, the VR program lost out on the ability to engage and serve these individuals, further contributing to the downward trends in engagement and programmatic outcomes nationally.

In terms of disability status, those living with psychosocial/psychological disabilities accounted for the highest number (33\%) of cases closed with the reason of Unable to Locate. All other disabilities had three or less cases based on review of VRC case notes. Specific disability types varied for those with psychosocial/psychological disabilities, with three noting schizophrenia, four noting severe depression and bipolar disorder, and one noting ADHD affecting their ability to obtain and/or maintain employment at application.

\section{Recommendations and Implications for Practice}

This study provided an opportunity to better understand the primary reasons for VR case closure following application, but prior to plan development; identify which disability groups are impacted; and identify discrepancies between coding in the state VR agencies' data system and actual reasons for case closure. Recently, TWC-VR services implemented enhancements to their case management system requiring staff to enter more thorough information regarding why the closure reason of All Other Reasons was the most appropriate selection instead of another code. This may help staff improve accuracy in noting and coding, with the aim of reducing the number of unnecessary premature case closures.

While there are many reasons why a case may be closed following application, there is room for improvement for VR agencies to effectively monitor this area, improve engagement with individuals, and ensure those with the most significant disabilities are served efficiently. Based on findings from this study, the following recommendations are offered:

- Data in the VR case file management system should be routinely reviewed for quality and used to inform opportunities for improving consumer communication and engagement and reducing early case closures.

- Provide training to ensure VR staff have a clear understanding of established case closure procedures and expectations, coding, and case noting requirements.

- Provide tools for VR staff to efficiently track cases in application status, with the aim of improving consumer communication, engagement, and retention.

- VR staff may benefit from additional training regarding the provision of general work incentives and employment support information to SSDI/SSI beneficiaries and recipients at the time of application.

- Future evaluation questions may consider exploring applicants' and community partner understanding of VR as an employment program designed to serve those interested in obtaining competitive, integrated employment. 


\section{Author Note}

The contents of this paper were developed under a cooperative agreement with the U.S. Department of Education, Technical Assistance Center for Vocational Rehabilitation Agency Program Evaluation and Quality Assurance (PEQA-TAC) (Grant Award Number: H263B150004). How- ever, the contents and views expressed in this publication do not necessarily represent the positions or policies of the U.S. Department of Education, and you should not assume endorsement by the Federal government. 


\section{References}

Houtenville, A., \& Rafal, M. (2020). Annual report on people with disabilities in America: 2020. University of New Hampshire, Institute on Disability. https://eric.e d.gov/?id=ED613092

Ipsen, C., \& Swicegood, G. (2014). Rural and urban differences in VR caseloads and delivery practices. The University of Montana Rural Institute. https://scholar works.umt.edu/ruralinst_employment/21/

Migliore, A., \& Landa, C. (2017). Reasons for exiting VR services without employment. University of Massachusetts Boston, Institute for Community Inclusion. https://scholarworks.umb.edu/thinkwork/9 $1 /$

Park, H., Kim-Rupnow, W., Stodden, R., \& Starbuck, D. (2005). Disparity of closures types in vocational rehabilitation services. Journal of Vocational Rehabilitation, 23(1), 33-38.

Pope, C. (2020, November 3). Data collection and analysis unit update and VR program data snapshot. 13th Annual Summit on Performance Management Excellence, Online.
Rehabilitation Services Administration. (n.d.). RSA-911 PD 16-04. U.S. Department of Education. https://www 2.ed.gov/policy/speced/guid/rsa/subregulatory/pd-1 6-04.pdf

Rogers, N. L., Embree, J., Masoudi, N., Huber, M., Ford, J., \& Moore, D. (2011). Consumer perspectives on reasons for unsuccessful VR case closure: An exploratory study. Journal of Vocational Rehabilitation, 34(3), 151-161. https://doi.org/10.3233/IVR-2011-05 $\underline{43}$

RTI International. (2003). Longitudinal study of the Vocational Rehabilitation Services program [Final report]. https://www2.ed.gov/rschstat/eval/rehab/vr-f inal-report-1.pdf

Texas Workforce Commission-Vocational Rehabilitation Division. (2020). Vocational Rehabilitation Services manual: VRSM B-600: Closure and post-employment services. Author. 